\title{
Ambulatory Hypertension in Pediatric Patients With Sickle Cell Disease and Its Association With End-Organ Damage
}

\author{
Saritha Ranabothu ${ }^{1}$, Michael Hafeman ${ }^{2}$, Deepa Manwani ${ }^{3}$, Kimberly Reidy ${ }^{4}$, Kerry Morrone ${ }^{3}$, \\ Josemiguel Lorenzo $^{5}$, Barbara Tria ${ }^{5}$, Frederick Kaskel ${ }^{4}$, Joseph Mahgerefteh ${ }^{5}$ \\ 1. Pediatrics, University of Arkansas for Medical Sciences, Little Rock, USA 2. Pediatrics, Albert Einstein College of \\ Medicine, Bronx, USA 3. Hematology, Children's Hospital at Montefiore, Bronx, USA 4. Nephrology, Children's \\ Hospital at Montefiore, Bronx, USA 5. Cardiology, Children's Hospital at Montefiore, Bronx, USA
}

Corresponding author: Saritha Ranabothu, sranabothu@uams.edu

\section{Abstract \\ Background}

Sickle cell disease (SCD), a chronic hemolytic disorder, results in cumulative end-organ damage affecting major organs such as the cardiovascular, renal, and central nervous systems. Effects of modifiable risk factors, such as blood pressure (BP), on the development of end-organ complications in SCD have not been well studied, particularly among the pediatric population. Relative hypertension in patients with SCD increases their risks of stroke, cardiovascular complications, and death. The primary hypothesis of this study was that abnormal BP patterns are common among patients with SCD and they impact end-organ complications.

\section{Methods}

Patients with SCD (HbSS, HbS $\beta$ ) were enrolled from the Children's Hospital at Montefiore ( $N=100)$. For each patient, demographic data were collected, biochemical variables in urine and blood samples were analyzed, BP was determined with ambulatory blood pressure monitoring (ABPM), and an echocardiogram was performed. The prevalence of abnormalities in BP parameters was defined, and their relationships with measures of SCD severity and end-organ damage were assessed.

\section{Results}

Sufficient ABPM data were available for 67 patients. Enrolled children were $13 \pm 4$ years ( $40 \%$ were males). Assessment of diurnal variation demonstrated that $81 \%$ of patients had abnormal systolic nocturnal dipping and $61 \%$ had abnormal diastolic nocturnal dipping. Abnormalities in the diurnal pattern were associated with reticulocytosis and hyperfiltration. Microalbuminuria was present in $19 \%(\mathrm{n}=13)$ of patients, of which $77 \%(n=10)$ were females $(p=0.014)$. Diastolic load and abnormal nocturnal dipping were associated with hyperfiltration but not with microalbuminuria.

Review began 10/28/2020 Review ended 11/16/2020 Published 11/25/2020

\section{(๑) Copyright 2020}

Ranabothu et al. This is an open access article distributed under the terms of the Creative Commons Attribution License CC-BY 4.0., which permits unrestricted use, distribution, and reproduction in any medium, provided the original author and source are credited.

\section{Conclusions}

BP abnormalities detected with ABPM in SCD patients are prevalent and perhaps are a risk factor for endorgan complications. Further studies are required to identify the mechanisms underlying these relationships and their longitudinal changes.

Categories: Pediatrics, Nephrology, Hematology

Keywords: sickle cell disease, hypertension, ambulatory blood pressure monitoring, end-organ complications, estimated glomerular filtration rate, microalbuminuria

\section{Introduction}

Sickle cell disease (SCD) is the most common inherited disorder of red blood cells in the United States [1]. Mortality and morbidity from SCD occur due to effects on major organ systems, including cardiovascular, renal, and central nervous systems [2]. Sickle cell nephropathy is a major complication of SCD that can result in significant mortality and morbidity. It is a progressive disease with varied manifestations, such as hyperfiltration, hypertrophy, and urine-concentrating defect. Sickle cell nephropathy can progress to microalbuminuria during childhood and, by adulthood, to macroalbuminuria and decline in renal function [3].

In general, hypertension is the most important modifiable risk factor for cardiovascular and renal disease [4]. Patients with SCD are known to have lower blood pressure (BP), which is due to unexplained etiology [5], but a high prevalence of masked hypertension and abnormal circadian patterns observed from ambulatory blood pressure monitoring (ABPM) among these patients has recently gained attention [6-8]. Variability in BP and 
loss of nocturnal dipping of BP in patients with SCD is associated with microalbuminuria and declines in renal function in relatively small studies by other investigators [7]. Our hypothesis is that abnormal circadian patterns and masked hypertension seen on ABPM are independently related to microalbuminuria and hyperfiltration. This hypothesis was tested, and the relationship between cardiovascular complications (ejection fraction, left ventricular mass index) and BP was assessed.

\section{Materials And Methods Study participants and study design}

Clinically stable children (age: $5-21$ years; $N=100$ ) with SCD from a pediatric sickle cell clinic at Children's Hospital at Montefiore, Albert Einstein College of Medicine, were enrolled from November 2015 until October 2017. The study was approved by the Institutional Review Board of Children's Hospital at Montefiore. Inclusion criteria were a diagnosis of SCD (HbSS, HbS $\beta 0$ ), confirmed with hemoglobin electrophoresis. Exclusion criteria were recent hospitalization, pain crisis within the prior 14 days, ongoing treatment with an antihypertensive agent, and known chronic kidney or congenital heart disease. Patients or guardians provided written informed assent/consents. Patients $\geqslant 18$ years were allowed to consent, those 1318 years were allowed to assent with legal guardian consent, and parents provided consent for children under the age of 13 years.

Baseline characteristics of each participant, including age, gender, weight, height, and BP, and comorbidities such as sleep apnea, were recorded. Patients underwent 24-hour ABPM with a Spacelabs 90207 model monitor, which is compact and lightweight and has five cuff sizes available for accuracy. A trained nurse or trained pediatric nephrology fellow placed and programed ABPMs. An appropriate-sized cuff was attached to the patient's non-dominant arm, and the device was programmed to record BP every 20 minutes during the daytime and every 30 minutes during nighttime. A study was considered interpretable when at least one BP reading per hour was valid and $>25$ readings in a 24 -hour period were valid. The same day that an ambulatory BP monitor was placed, blood and urine samples were collected and analyzed for complete blood count, creatinine, lactate dehydrogenase (LDH), reticulocyte count, fetal hemoglobin, urine microalbumin, and urine creatinine. Estimated glomerular filtration rate (eGFR) was measured with the modified Schwartz formula for patients $<18$ years old [9] and with the Modification of Diet in Renal Disease formula for patients $\geqslant 18$ years old [10].

BP load, pre-hypertension, hypertension, and masked hypertension were defined according to the most recent ABPM statement issued by the American Heart Association [11]. BPs in the clinic were defined according to the 2017 consensus guidelines of the American Academy of Pediatrics [12].

Adequate nocturnal dipping was defined as at least a $10 \%$ decrease, relative to diurnal BP, in nocturnal systolic and diastolic BP. Blunted dipping was defined as $<10 \%$ drop in nocturnal systolic or diastolic BP, extreme dipping as $>20 \%$ drop in nocturnal systolic or diastolic BP, and reverse dipping as sleep systolic or diastolic BP higher than awake systolic or diastolic BP.

The systolic BP index was calculated as the measured mean BP divided by the 95th percentile for sex and height [13]. Hyperfiltration was defined as eGFR $>180 \mathrm{~mL} / \mathrm{min} / 1.73 \mathrm{~m}^{2}$ for patients $<10$ years old and as $>140$ $\mathrm{mL} / \mathrm{min} / 1.73 \mathrm{~m}^{2}$ for patients $\geqslant 10$ years old [14]. Microalbuminuria was defined as urinary albumin to creatinine ratio between 30 and $300 \mathrm{mg} / \mathrm{g}$ [15].

\section{Echocardiogram}

Echocardiograms were obtained at the scheduled time annually. If echocardiogram results were more than one year old at the time of enrollment, then a repeated study was requested. All echocardiograms were performed according to the institutional protocol and in accordance with the American Society of Echocardiography published guidelines on performing and quantifying pediatric echocardiograms [16].

\section{Data analysis}

Descriptive statistics (mean \pm standard deviation) were generated for all demographic characteristics. All values were checked for normality. Means, confidence intervals, standard deviations, standard errors, ranges, $\mathrm{p}$-values, and equality of variances were calculated for all variables. Demographic information, anthropomorphic data, and all echocardiographic measurements and calculations were compared between patients with and without hypertension.

Pearson and Spearman correlation and multivariate regression analyses were performed. All tests were twosided. Statistical significance was defined as $\mathrm{p}<0.05$. Statistical analyses were performed with SPSS Version 22 (IBM Corp., Armonk, NY, USA).

\section{Results}

Of 100 patients who were enrolled, 67 (40\% male) were included in the analysis; the mean age was $13 \pm 4$ 


\section{Cureus}

years old. Thirty-three patients were excluded due to the lack of sufficient data on ABPM $(n=29)$ or because a pain crisis occurred after enrollment $(n=4)$. Patients with no echocardiogram within one year of ABPM or insufficient echocardiogram were excluded from the cardiac analyses $(\mathrm{n}=21)$ (Figure 1).

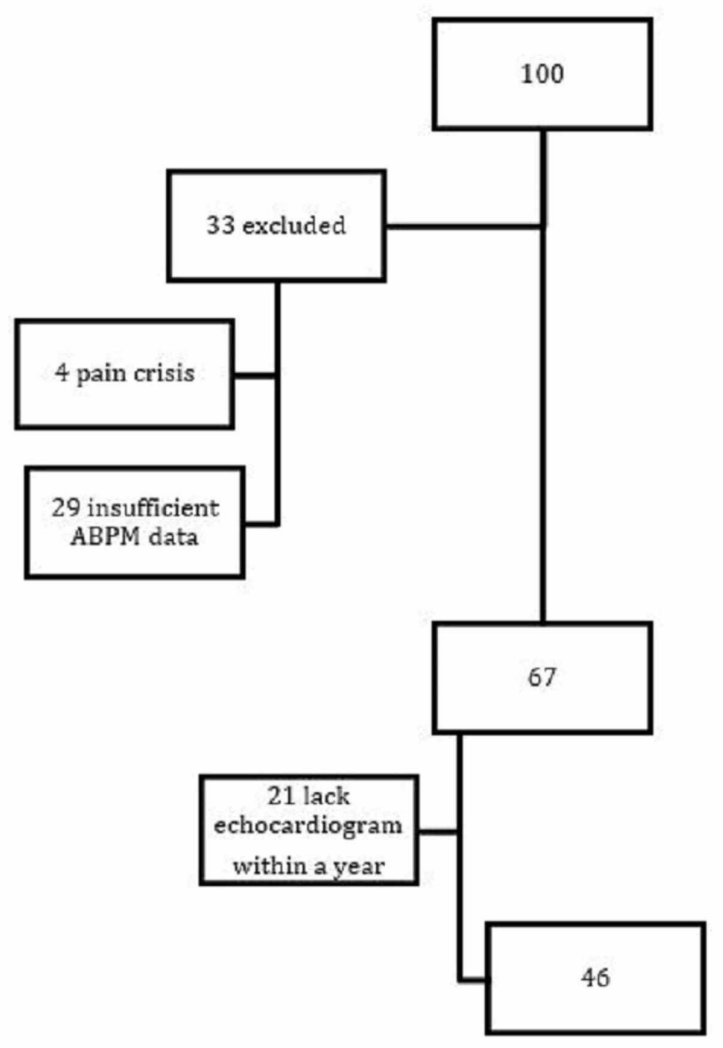

\section{FIGURE 1: Patient cohort}

A total of 100 patients were enrolled; 33 patients were subsequently excluded due to pain crisis or insufficient ABPM data. Of the remaining 67 patients, 21 lacked an echocardiogram within one year of ABPM; thus, only 48 patients were included for correlation analyses of cardiovascular complications.

ABPM, ambulatory blood pressure monitoring

Clinical characteristics and biochemical variables are shown in Table 1. None of the patients were obese. 


\section{Cureus}

\begin{tabular}{ll}
\hline Age $($ mean \pm SD) & $13 \pm 4$ years \\
Sex & $40 \%$ male \\
Race & $11 \%$ Hispanic \\
BMI z-score $\left(\mathrm{kg} / \mathrm{m}^{2}\right)$ & $-0.3 \pm 1.3$ \\
VOC & $29 \%$ \\
ACS & $16 \%$ \\
Hydroxyurea & $72 \%$ \\
Pain medications & $7 \%$ \\
FHx of HTN & $17 \%$ \\
Hb $(\mathrm{g} / \mathrm{dL})$ & $8.7 \pm 1.329 \%$ \\
eGFR $(\mathrm{mL})$ & $136 \pm 38$ \\
LDH $(\mathrm{U} / \mathrm{mL})$ & $468 \pm 171$ \\
Urine microalbumin $(\mathrm{mg} / \mathrm{dL})$ & $28 \pm 46$ \\
Retic $\%$ & $11 \pm 6$ \\
HbF $\%$ & $11.5 \pm 8.6$
\end{tabular}

\section{TABLE 1: Clinical characteristics ( $N=67$ )}

BMI, body mass index; VOC, vaso-occlusive crisis (painful crisis caused by ischemic tissue injury); ACS, acute chest syndrome (acute lung injury, represents lung infarction, inflammation, atelectasis); FHx of HTN, family history of hypertension; Hb, hemoglobin; eGFR, estimated glomerular filtration rate; $\mathrm{LDH}$, lactate dehydrogenase; Retic, reticulocyte; $\mathrm{HbF}$, fetal hemoglobin

ABPM demonstrated that $7.5 \%(\mathrm{n}=5)$ were hypertensive (three with masked hypertension, two with ambulatory hypertension), and $9 \%(\mathrm{n}=6)$ were pre-hypertensive (Figure 2$)$.

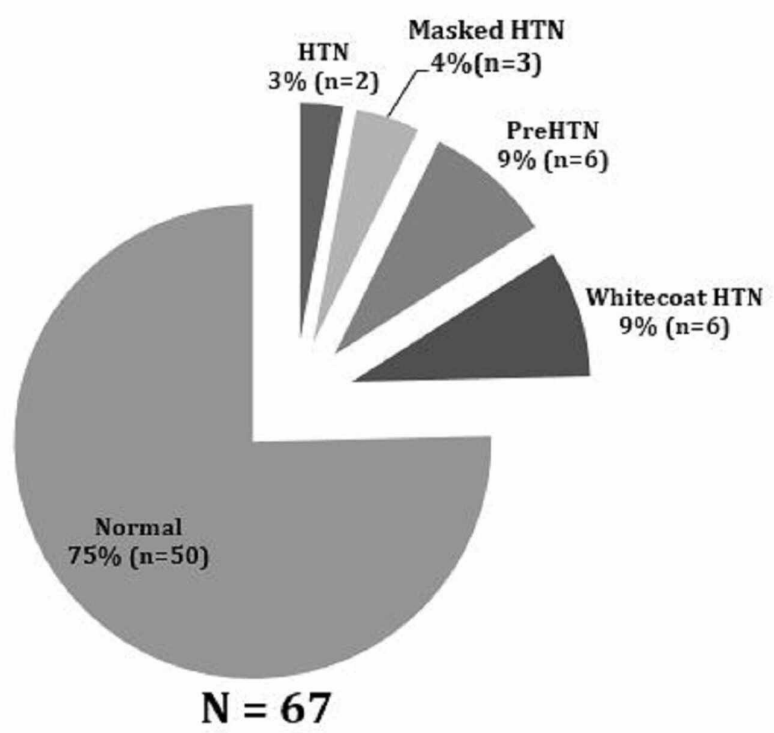

FIGURE 2: Percentage of patients with HTN

HTN, hypertension 


\section{Cureus}

Abnormal systolic nocturnal dipping was present in 81\% $(n=53)$; of these, $10 \%(n=7)$ had reverse systolic dipping (Figure 3).

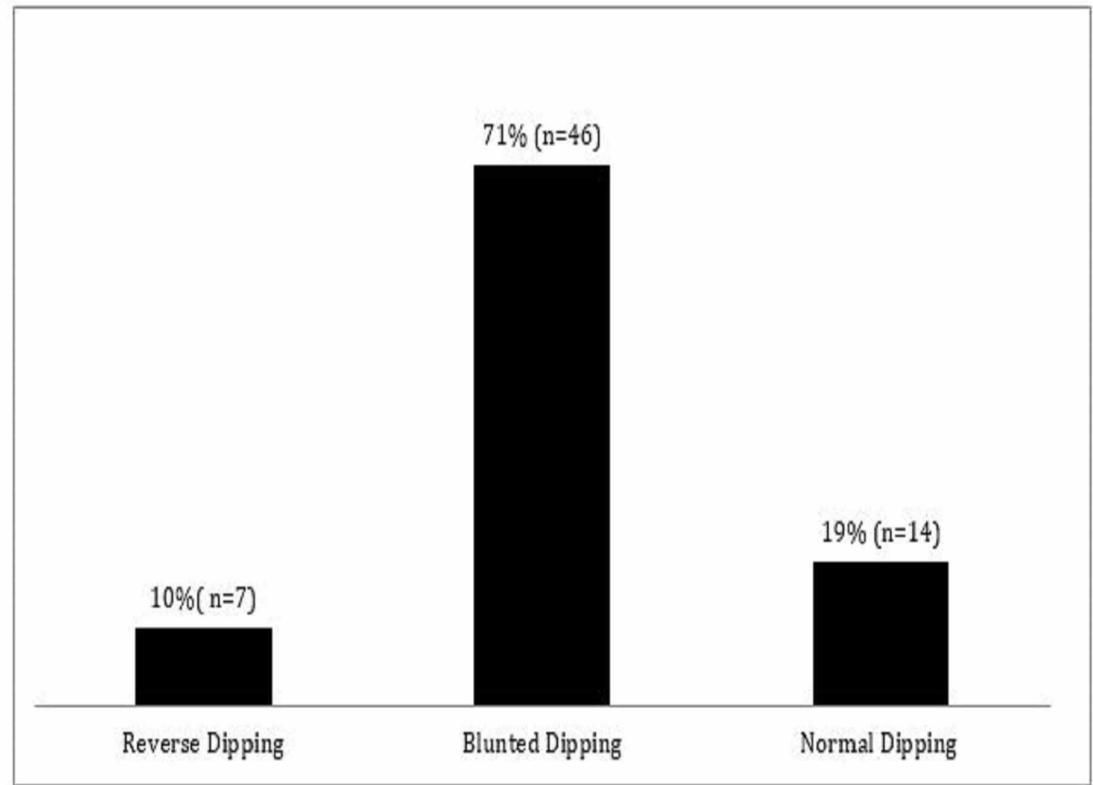

FIGURE 3: Prevalence of systolic nocturnal dipping abnormalities

Abnormal diastolic nocturnal dipping was present in 61\% ( $n=42)$; $9 \%$ had reverse dipping and $9 \%$ had extreme diastolic nocturnal dipping. Abnormal nocturnal dipping and hypertension were noted across all age groups. BP parameters were not associated with low hematocrit (hct), markers of inflammation (white blood cell count [WBC], absolute neutrophil count [ANC] [10,17]), or markers of hemolysis (LDH, bilirubin, aspartate aminotransferase).

Echocardiograms demonstrated that $61 \%$ of patients (30/49) had left ventricle (LV) dilation, but all had a normal systolic function, assessed by ejection fraction, and there was no evidence of significant pulmonary hypertension. Correlations between echocardiographic findings and laboratory hematologic findings are shown in Table 2.

\begin{tabular}{|c|c|c|c|c|c|c|c|c|c|c|}
\hline & \multicolumn{2}{|l|}{ Het } & \multicolumn{2}{|c|}{ Retic \% } & \multicolumn{2}{|l|}{ LDH } & \multicolumn{2}{|l|}{ WBC } & \multicolumn{2}{|l|}{ ANC } \\
\hline & $r$ & $p$-Value & $r$ & p-Value & $r$ & p-Value & $r$ & $p$-Value & $r$ & p-Value \\
\hline EDV z-score & -0.41 & $<0.001$ & 0.36 & 0.001 & 0.4 & $<0.001$ & 0.3 & 0.006 & 0.13 & 0.24 \\
\hline LVMI & -0.30 & 0.005 & 0.38 & 0.007 & 0.28 & 0.011 & 0.28 & 0.009 & 0.22 & 0.05 \\
\hline LV M/V ratio z-score & 0.173 & 0.11 & -0.15 & 0.17 & -0.13 & 0.22 & -0.07 & 0.537 & -0.07 & 0.54 \\
\hline
\end{tabular}

TABLE 2: Correlations between echocardiographic findings and laboratory hematologic findings

Hct, hematocrit; Retic, reticulocyte; LDH, lactate dehydrogenase; WBC, white blood cell count; ANC, absolute neutrophil count; EDV, end diastolic volume; LVMI, left ventricular mass index; LV M/V ratio, left ventricular mass-to-volume ratio

As expected, results showed that left ventricular mass volume index (LVMI) and end-diastolic volume (EDV) were significantly related to hct, hemolysis (LDH), and inflammation (WBC, ANC). Correlations between ambulatory BP variables and echocardiographic markers of size and systolic function were not significant except for diastolic load, diastolic nocturnal dipping, and 24-hour diastolic BP variability (diastolic load and mitral valve $S^{\prime} r=-0.3, p=0.03$; diastolic nocturnal dipping and ejection fraction $\mathrm{z}$-score $\mathrm{r}=-0.2, \mathrm{p}=0.04$; 24-hour diastolic variability and left ventricular mass-to-volume ratio $z$-score $r=0.3, p=0.02 ; 24$-hour diastolic variability and mitral valve $S^{\prime} r=-0.3, p=0.02$ ). 


\section{Cureus}

Correlations between markers of renal function and laboratory hematologic findings are shown in Table 3.

\begin{tabular}{|c|c|c|c|c|c|c|c|c|}
\hline & \multicolumn{2}{|l|}{ Hct } & \multicolumn{2}{|c|}{ Retic \% } & \multicolumn{2}{|l|}{ LDH } & \multicolumn{2}{|l|}{ WBC } \\
\hline & $r$ & p-Value & $r$ & p-Value & $r$ & p-Value & $r$ & p-Value \\
\hline eGFR & -0.45 & $<0.001$ & 0.48 & $<0.001$ & 0.52 & $<0.001$ & 0.26 & 0.01 \\
\hline Creatinine & 0.40 & $<0.001$ & -0.45 & $<0.001$ & -0.52 & $<0.001$ & -0.26 & 0.01 \\
\hline Urine MA/Cr & -0.16 & 0.17 & 0.35 & 0.001 & 0.22 & 0.054 & 0.30 & 0.005 \\
\hline
\end{tabular}

\section{TABLE 3: Correlation between hemolysis and renal function}

Hct, hematocrit; Retic, reticulocyte; LDH, lactate dehydrogenase; WBC, white blood cell count; eGFR, estimated glomerular filtration rate; urine $\mathrm{MA} / \mathrm{Cr}$; urine microalbumin-to-creatinine ratio

Hyperfiltration was related to hct, hemolysis (LDH), and inflammation (WBC). In addition, protein in urine was significantly related to hemolysis (LDH) and inflammation (WBC). Correlations between markers of renal function and ambulatory BP variables are shown in Table 4.

\begin{tabular}{|c|c|c|c|c|c|c|c|c|c|c|c|c|c|c|}
\hline & \multicolumn{2}{|c|}{ Sys ND } & \multicolumn{2}{|c|}{ Dia ND } & \multicolumn{2}{|c|}{ 24-h sys BP var } & \multicolumn{2}{|c|}{ 24-h dia BP var } & \multicolumn{2}{|c|}{ Aver HR } & \multicolumn{2}{|c|}{$\%$ Sys load } & \multicolumn{2}{|c|}{ \% Dia load } \\
\hline & $r$ & p-Value & $r$ & p-Value & $r$ & $\mathrm{p}$-Value & $r$ & p-Value & $r$ & p-Value & $r$ & $p$-Value & $r$ & p-Value \\
\hline eGFR & -0.21 & 0.06 & -0.25 & 0.02 & -0.02 & 0.83 & -0.22 & 0.05 & 0.32 & 0.008 & 0.12 & 0.3 & 0.25 & 0.03 \\
\hline Creatinine & 0.12 & 0.30 & 0.15 & 0.19 & 0.07 & 0.55 & 0.25 & 0.02 & -0.4 & 0.001 & -0.03 & 0.81 & -0.17 & 0.13 \\
\hline Urine MA/Cr & -0.18 & 0.08 & -0.13 & 0.2 & 0.01 & 0.73 & -0.03 & 0.94 & 0.05 & 0.72 & 0.06 & 0.64 & -0.05 & 0.68 \\
\hline
\end{tabular}

\section{TABLE 4: Correlation between ambulatory blood pressure and renal function}

Sys ND, systolic nocturnal dipping; Dia ND, diastolic nocturnal dipping; 24-h sys BP var, 24-hour systolic blood pressure variability; 24-h dia BP var, 24-hour diastolic blood pressure variability; Aver HR, average heart rate; Sys load, systolic load; Dia load, diastolic load; eGFR, estimated glomerular filtration rate; urine $\mathrm{MA} / \mathrm{Cr}$, urine microalbumin-to-creatinine ratio

Hyperfiltration was related to higher diastolic load, worse nocturnal dipping, and higher average heart rate. Relationships between markers of renal function and echocardiographic findings are shown in Table 5.

\begin{tabular}{|c|c|c|c|c|c|c|c|c|c|c|}
\hline & \multicolumn{2}{|c|}{ EDV Z score } & \multicolumn{2}{|l|}{ LVMI } & \multicolumn{2}{|c|}{ LV M/V ratio Z score } & \multicolumn{2}{|c|}{ EF Z score } & \multicolumn{2}{|l|}{ MV S' } \\
\hline & $r$ & $p$-Value & $r$ & p-Value & $r$ & $\mathrm{p}$-Value & $r$ & $p$-Value & $r$ & p-Value \\
\hline eGFR & 0.25 & 0.02 & 0.17 & 0.12 & -0.22 & 0.05 & 0.23 & 0.04 & 0.02 & 0.84 \\
\hline Creatinine & -0.30 & 0.009 & -0.11 & 0.31 & 0.23 & 0.04 & -0.27 & 0.01 & -0.002 & 0.99 \\
\hline Urine MA/Cr & 0.02 & 0.81 & -0.007 & 0.95 & -0.14 & 0.23 & 0.10 & 0.41 & 0.01 & 0.88 \\
\hline
\end{tabular}

\section{TABLE 5: Correlation between renal function and echocardiogram findings}

EDV, end-diastolic volume; LVMI, left ventricular mass index; LV M/V ratio, left ventricular mass-to-volume ratio; EF, ejection fraction; MV S', peak velocity of systolic mitral valve annular motion; eGFR, estimated glomerular filtration rate; urine MA/Cr, urine microalbumin-to-creatinine ratio

Hyperfiltration was significantly related to higher left ventricular mass and lower mass-to-volume ratio. Renal function was positively correlated with left ventricular systolic function.

Microalbuminuria was present in $19 \%$ of patients $(n=13)$, of whom $77 \%(n=10)$ were female $(p=0.014)$. Of the 28 patients who had a sleep study, eight (28.5\%) were found to have sleep apnea. All but one of the 
patients with sleep apnea had either blunted or reverse systolic nocturnal dipping (average systolic dipping: 4.25\%; diastolic dipping: $8.5 \%$ ). eGFR was $>90 \mathrm{~mL} / \mathrm{min} / 1.73 \mathrm{~m}^{2}$ for all but one of the patients; the patient was not hypertensive, did not have microalbuminuria, and had normal echocardiograms. There was no significant difference in microalbuminuria or eGFR in patients who were on hydroxyuria versus those who were not.

\section{Discussion}

Abnormal nocturnal dipping was seen in $81 \%$ of patients; of those, $10 \%$ had reverse dipping. BP follows a circadian rhythm; levels rise during daytime or with activity and fall during nighttime or with sleep. Abnormal circadian patterns are associated with renal and cardiovascular complications in patients with chronic kidney disease or diabetes, and even within the healthy population [18-20]. Our results confirmed previous reports of a high prevalence of ABPM abnormalities, especially abnormal nocturnal dipping, in pediatric patients with SCD [6-8].

The overall rate of ambulatory hypertension of 7.42\% (5/67) observed in this study population is comparable to reported prevalence in the general population of pediatric patients [21-22]. In addition, six patients were pre-hypertensive. Shatat et al. [8] reported ambulatory hypertension in $44 \%(\mathrm{n} / \mathrm{N}, 17 / 38)$ of their study population; 34\% (13/38) had masked hypertension, 59\% had impaired systolic nocturnal dipping, and 18\% had impaired diastolic nocturnal dipping. In another study, Becker et al. obtained ABPM data on 52 pediatric patients with SCD, which showed that 35\% had masked hypertension, $17 \%$ had pre-hypertension, and 56\% had abnormal nocturnal dipping [7]. In the most recent study, Moodalbail et al. reported ABPM data on 56 pediatric patients with SCD that showed $30 \%(n=17)$ were hypertensive; 3 had ambulatory hypertension and 14 had masked hypertension [6]. Half of the patients had pre-hypertension/abnormal nocturnal dipping [6]. Overall, in our study population, the prevalence of hypertension/masked hypertension was lower, but rates of blunted nocturnal dipping were higher. The higher rate of blunted dipping in our study may be due, in part, to differences in the type of SCD. For example, most patients in our study population have severe genotypes of SCD, but almost one-third of the patients in the study conducted by Shatat et al. had sickle cell trait (HbSC) [8].

Previous studies have proposed adding ABPM as a standard of care for all patients with SCD [8]. Together, our results and those from other studies support a role for 24-hour ABPM in all pediatric SCD patients and suggest that SCD may be a condition that, similar to chronic kidney disease, increases risk of masked hypertension. Importantly, the Centers for Medicare \& Medicaid Services recently finalized a national coverage policy for ABPM. SCD was not specified in the policy, but it does specify that routine ABPM be considered in "children with high-risk conditions." Similarly, SCD was not listed as a high-risk condition when the American Academy of Pediatrics recently issued its clinical practice guidelines on screening and managing hypertension in children and adolescents [12]. Our results and those of others support the inclusion of SCD as a high-risk condition.

Consistent with other studies, 19\% ( $n=13)$ of patients in our study had microalbuminuria, most of whom were females (11/13). Abnormal dipping in patients with microalbuminuria did not differ from that in patients without microalbuminuria, which is inconsistent with the results of previous studies. In concordance with previous studies, most (49\%) of our study population had hyperfiltration [23]. Previous reports showed a mean eGFR of around $155 \pm 40 \mathrm{~mL} / \mathrm{min} / 1.73 \mathrm{~m}^{2}$ (measured with 99mTc-DTPA) in children with SCD who were $<16$ years of age; it was shown to decline during the second decade of life, with a mean eGFR of around $133 \pm 31 \mathrm{~mL} / \mathrm{min} / 1.73 \mathrm{~m}^{2}$ in children $>16$ years of age [24]. In the present study, the mean eGFR $\left(136 \pm 38 \mathrm{ml} / \mathrm{min} / 1.73 \mathrm{~m}^{2}\right)$ was lower than in the previous studies [24-25]. Of note, 28 (40\%) out of 69 patients had eGFR $<130 \mathrm{~mL} / \mathrm{min} / 1.73 \mathrm{~m}^{2}$, as determined by serum creatinine measurements. Low eGFR was not associated with high WBC, high LDH, low hemoglobin, or nocturnal hypertension, as was demonstrated in the past [26]. Hyperfiltration, however, was related to inflammation and hemolysis. Most patients (20 out of $28 ; 71 \%$ ) with low eGFR ( $<130 \mathrm{~mL} / \mathrm{min} / 1.73 \mathrm{~m}^{2}$ ) were on hydroxyurea. The etiology of lower mean eGFR observed in this study, as compared to published studies, is unclear but may be related to the differences in measured 99mTc-DTPA versus calculated eGFR. Further studies are needed to establish the causal relationship and potential benefit of treating diastolic nocturnal dipping and diastolic load to slow the progression of sickle cell nephropathy.

Many studies of adults showed that SCD is associated with elevated cardiac output, cardiomegaly, cardiac dysfunction/diastolic dysfunction, and pulmonary hypertension [27-28]. In our study of a pediatric population, left ventricular dilation was seen in $61 \%$ of patients, but ventricular function was normal, there was no evidence of significant pulmonary hypertension, and systolic nocturnal dipping was not associated with cardiac remodeling. However, diastolic nocturnal dipping, diastolic load, and diastolic BP variability negatively impact left ventricular remodeling and systolic function. A previous study showed that anemia and hemoglobin concentration have a relationship with LV size [29]. As expected, our study demonstrated that cardiac size has a significant relationship with anemia, hemolysis, and inflammation. In addition, reticulocytosis was significantly correlated with ejection fraction, which may warrant further investigation. 


\section{Limitations}

Conclusions drawn from the results of this study are limited by the study's small size and cross-sectional design. All enrolled patients could not be included in the analysis because some did not have valid ABPM results or interpretable echocardiograms. Patients were included if they had at least 25 ABPM readings, which is fewer than the recommended number of measurements in the research. General limitations of ABPM that may affect measurements and detection of nocturnal abnormalities include differences between bedtime and actual sleep time, as well as discomfort due to the monitor; however, these limitations are likely to impact our study to the same extent that they impact others that have used ABPM. Data were not reproduced. Microalbuminuria was determined from a single random sample. Also, the use of creatinine to calculate eGFR may affect results. Finally, $23 \%$ of patients were on chronic transfusion, and some received $\mathrm{ABPM}$ on the last day of transfusion or one day after the completion of transfusion, which may affect hemoglobin and markers of hemolysis.

\section{Conclusions}

Abnormal BP patterns were noted for patients with SCD, and accurate diagnosis of most of these abnormalities requires ABPM. Thus, the use of ABPM in this at-risk population may be warranted. SCD patients have other risk factors, such as hemolysis, vaso-occlusion, and chronic inflammation, that could contribute to end-organ complications. However, patients who are at high risk of sustained hypertension (i.e., those with pre-hypertension or masked hypertension) need to be assessed and treated carefully to lessen end-organ damage. Long-term follow-up studies are needed to assess the role of abnormal nocturnal dipping in stroke, cardiovascular, or renal complications.

\section{Additional Information \\ Disclosures}

Human subjects: Consent was obtained by all participants in this study. Institutional Review Board of Children's Hospital at Montefiore, Albert Einstein College of Medicine issued approval 2015-5306. Animal subjects: All authors have confirmed that this study did not involve animal subjects or tissue. Conflicts of interest: In compliance with the ICMJE uniform disclosure form, all authors declare the following: Payment/services info: The project was supported in part by the National Center for Advancing Translational Sciences (NCATS), components of the National Institutes of Health (NIH), through CTSA grant number UL1TR001073. Its contents are solely the responsibility of the authors and do not necessarily represent the official views of the NIH. . Financial relationships: All authors have declared that they have no financial relationships at present or within the previous three years with any organizations that might have an interest in the submitted work. Other relationships: All authors have declared that there are no other relationships or activities that could appear to have influenced the submitted work.

\section{References}

1. Hassell KL: Population estimates of sickle cell disease in the U.S . Am J Prev Med. 2010, 38:512-21. 10.1016/j.amepre.2009.12.022

2. Steinberg MH: Sickle cell anemia, the first molecular disease: overview of molecular etiology, pathophysiology, and therapeutic approaches. ScientificWorldJournal. 2008, 8:1295-324. 10.1100/tsw.2008.157

3. Nath KA, Hebbel RP: Sickle cell disease: renal manifestations and mechanisms. Nat Rev Nephrol. 2015, 11:161-71. 10.1038/nrneph.2015.8

4. Botdorf J, Chaudhary K, Whaley-Connell A: Hypertension in Cardiovascular and Kidney Disease. Cardiorenal Med. 2011, 1:183-92. 10.1159/000329927

5. de Jong PE, Landman H, van Eps LW: Blood pressure in sickle cell disease . Arch Intern Med. 1982, 142:123940.

6. Moodalbail DG, Falkner B, Keith SW, Mathias RS, Araya CE, Zaritsky JJ, Stuart MJ: Ambulatory hypertension in a pediatric cohort of sickle cell disease. J Am Soc Hypertens. 2018, 12:542-50. 10.1016/j.jash.2018.04.005

7. Becker AM, Goldberg JH, Henson M, Ahn C, Tong L, Baum M, Buchanan GR: Blood pressure abnormalities in children with sickle cell anemia. Pediatr Blood Cancer. 2014, 61:518-22. 10.1002/pbc.24843

8. Shatat IF, Jakson SM, Blue AE, Johnson MA, Orak JK, Kalpatthi R: Masked hypertension is prevalent in children with sickle cell disease: a Midwest Pediatric Nephrology Consortium study. Pediatr Nephrol. 2013, 28:115-20. 10.1007/s00467-012-2275-9

9. Schwartz GJ, Muñoz A, Schneider MF, Mak RH, Kaskel F, Warady BA, Furth SL: New equations to estimate GFR in children with CKD. J Am Soc Nephrol. 2009, 20:629-37. 10.1681/ASN.2008030287

10. Levey AS, Bosch JP, Lewis JB, Greene T, Rogers N, Roth D: A more accurate method to estimate glomerular filtration rate from serum creatinine: a new prediction equation. Modification of Diet in Renal Disease Study Group. Ann Intern Med. 1999, 130:461-70. 10.7326/0003-4819-130-6-199903160-00002

11. Flynn JT, Daniels SR, Hayman LL, et al.: Update: ambulatory blood pressure monitoring in children and adolescents: a scientific statement from the American Heart Association. Hypertension. 2014, 63:1116-35. 10.1161/HYP.0000000000000007

12. Flynn JT, Falkner BE: New clinical practice guideline for the management of high blood pressure in children and adolescents. Hypertension. 2017, 70:683-6. 10.1161/HYPERTENSIONAHA.117.10050

13. Samuels J, Ng D, Flynn JT, Mitsnefes M, Poffenbarger T, Warady BA, Furth S; Chronic Kidney Disease in Children Study Group: Ambulatory blood pressure patterns in children with chronic kidney disease . 
Hypertension. 2012, 60:43-50. 10.1161/HYPERTENSIONAHA.111.189266

14. Hjorth L, Wiebe T, Karpman D: Hyperfiltration evaluated by glomerular filtration rate at diagnosis in children with cancer. Pediatr Blood Cancer. 2011, 56:762-6. 10.1002/pbc.22971

15. National Kidney Foundation: KDOQI Clinical Practice Guidelines and Clinical Practice Recommendations for Diabetes and Chronic Kidney Disease. Am J Kidney Dis. 2007, 49:12-154. 10.1053/j.ajkd.2006.12.005

16. Lopez L, Colan SD, Frommelt PC, et al.: Recommendations for quantification methods during the performance of a pediatric echocardiogram: a report from the Pediatric Measurements Writing Group of the American Society of Echocardiography Pediatric and Congenital Heart Disease Council. J Am Soc Echocardiogr. 2010, 23:465-95. 10.1016/j.echo.2010.03.019

17. Jodele S, Dandoy CE, Lane A, et al.: Complement blockade for TA-TMA: lessons learned from a large pediatric cohort treated with eculizumab. Blood. 2020, 135:1049-57. 10.1182/blood.2019004218

18. Velasquez MT, Beddhu S, Nobakht E, Rahman M, Raj DS: Ambulatory blood pressure in chronic kidney disease: ready for prime time?. Kidney Int Rep. 2016, 1:94-104. 10.1016/j.ekir.2016.05.001

19. Felicio JS, de Souza AC, Kohlmann N, Kohlmann O, Jr., Ribeiro AB, Zanella MT: Nocturnal blood pressure fall as predictor of diabetic nephropathy in hypertensive patients with type 2 diabetes. Cardiovasc Diabetol. 2010, 9:36. 10.1186/1475-2840-9-36

20. Gkaliagkousi E, Anyfanti P, Lazaridis A, et al.: Clinical impact of dipping and nocturnal blood pressure patterns in newly diagnosed, never-treated patients with essential hypertension. J Am Soc Hypertens. 2018, 12:850-7. 10.1016/j.jash.2018.08.004

21. Fan Z, Liao Z, Zong X, Zhang S: Differences in prevalence of prehypertension and hypertension in children and adolescents in the eastern, central and western regions of China from 1991-2011 and the associated risk factors. PLoS One. 2019, 14:0210591. 10.1371/journal.pone.0210591

22. Bell CS, Samuel JP, Samuels JA: Prevalence of hypertension in children. Hypertension. 2019, 73:148-52. 10.1161/HYPERTENSIONAHA.118.11673

23. Guasch A, Navarrete J, Nass K, Zayas CF: Glomerular involvement in adults with sickle cell hemoglobinopathies: Prevalence and clinical correlates of progressive renal failure. J Am Soc Nephrol. 2006, 17:2228-35. 10.1681/ASN.2002010084

24. Aygun B, Mortier NA, Smeltzer MP, Hankins IS, Ware RE: Glomerular hyperfiltration and albuminuria in children with sickle cell anemia. Pediatr Nephrol. 2011, 26:1285-90. 10.1007/s00467-011-1857-2

25. Bodas P, Huang A, O'Riordan MA, Sedor JR, Dell KM: The prevalence of hypertension and abnormal kidney function in children with sickle cell disease -a cross sectional review. BMC Nephrol. 2013, 14:237. 10.1186/1471-2369-14-237

26. Itano HA, Keitel HG, Thompson D: Hyposthenuria in sickle cell anemia: a reversible renal defect . J Clin Invest. 1956, 35:998-1007. 10.1172/JCI103360

27. Fitzhugh CD, Lauder N, Jonassaint JC, et al.: Cardiopulmonary complications leading to premature deaths in adult patients with sickle cell disease. Am J Hematol. 2010, 85:36-40. 10.1002/ajh.21569

28. Rees DC, Williams TN, Gladwin MT: Sickle-cell disease. Lancet. 2010, 376:2018-31. 10.1016/S01406736(10)61029-X

29. Lester LA, Sodt PC, Hutcheon N, Arcilla RA: Cardiac abnormalities in children with sickle cell anemia . Chest. 1990, 98:1169-74. 10.1378/chest.98.5.1169 